\title{
Cycle length alternation in supraventricular tachycardia after administration of verapamil
}

\author{
Jitu Vohra, David Hunt, John Stuckey, and Graeme Sloman \\ From the Cardiac Laboratory, Royal Melbourne Hospital, Victoria, Australia
}

Four patients are reported in whom alternation of $R R$ cycle length before reversion to sinus rhythm occurred during the administration of intravenous verapamil. In 2 of these 4 patients, alternation of cycle lengths was associated with a changing $Q R S$ morphology.

It is suggested that one patient had a reciprocating tachycardia using dual AV nodal pathways. The alternating cycle lengths in response to verapamil are explained on the basis of a $2: 1$ block in the antegrade pathway unmasking a third pathway, thus providing two pathways with differing antegrade conduction. In the second patient the mechanism of the supraventricular tachycardia appears to be atrial or $A V$ nodal extrasystolic tachycardia. We attribute the alternating cycle length to a 3:2 Wenckebach block before reversion to sinus rhythm. However, a reciprocating mechanism in this and the other two patients cannot be excluded. The possible mechanisms for verapamil-induced alternation are discussed.

Paroxysmal supraventricular tachycardia results either from a rapidly discharging ectopic pacemaker or through a re-entry mechanism (Katz and Pick, 1960). As a rule, the $R R$ cycles are regular, but alternating cycle lengths during supraventricular tachycardia have been reported (Barker, Johnston, and Wilson, 1943, Langendorf, 1958; Spurrell, Krikler, and Sowton, 1973). Alternation in extrasystolic atrial and AV junctional tachycardias can be explained on the basis of a 3:2 Wenckebach block. Moe, Childers, and Merideth (1968) have suggested that even in an extrasystolic tachycardia, the impulse is conducted through both AV nodal pathways and cycle length alternation may be explained on the basis of a 2:I block in one of the two pathways.

The generally accepted basis for a re-entrant reciprocating tachycardia is the existence of dual AV nodal pathways (alpha and beta) as suggested by Mendez and Moe (I966). Alternating RR cycle lengths in a sustained reciprocal tachycardia using only two pathways is difficult to explain. Schuilenberg and Durrer (I968) proposed a basis for the alternation of $R R$ cycle lengths by suggesting three pathways. The presence of multiple pathways with differing conduction and refractoriness has also been suggested (Watanabe and Dreifus, 1965). Spurrell et al. (1973) reported two cases of recip-

Received 29 October 1973. rocating tachycardias with alternating cycle lengths.

We report 4 patients in whom alternating cycle lengths occurred before the termination of supraventricular tachycardia during the administration of verapamil.

Verapamil administered by the intravenous route is of value in the treatment of supraventricular tachycardia. The mechanism of the antiarrhythmic action is not clear, but an increase in the atrioventricular conduction time induced by the drug is considered to play an important role (Schamroth, Krikler, and Garrett, 1972).

\section{Case I}

\section{Case reports}

A 54-year-old housewife was admitted with a three hour history of palpitations. She had experienced recurrent palpitations for three years and at the time of admission she was receiving $20 \mathrm{mg}$ propranolol four times a day. A regular heart rate of 160 a minute was recorded with a blood pressure of $135 / 105 \mathrm{mmHg}$. There was no evidence of cardiac failure. The electrocardiogram showed QRS complexes of normal width and regularly associated $P$ waves suggestive of a supraventricular tachycardia (Fig. I). The frontal $P$ wave axis could not be determined as the $P$ waves are superimposed on the T waves (Massumi and Desando, 1972).

The RR interval was regular at $380 \mathrm{msec}$. After the administration of $2 \mathrm{mg}$ verapamil by intravenous injection, alternation of QRS amplitude developed, though 


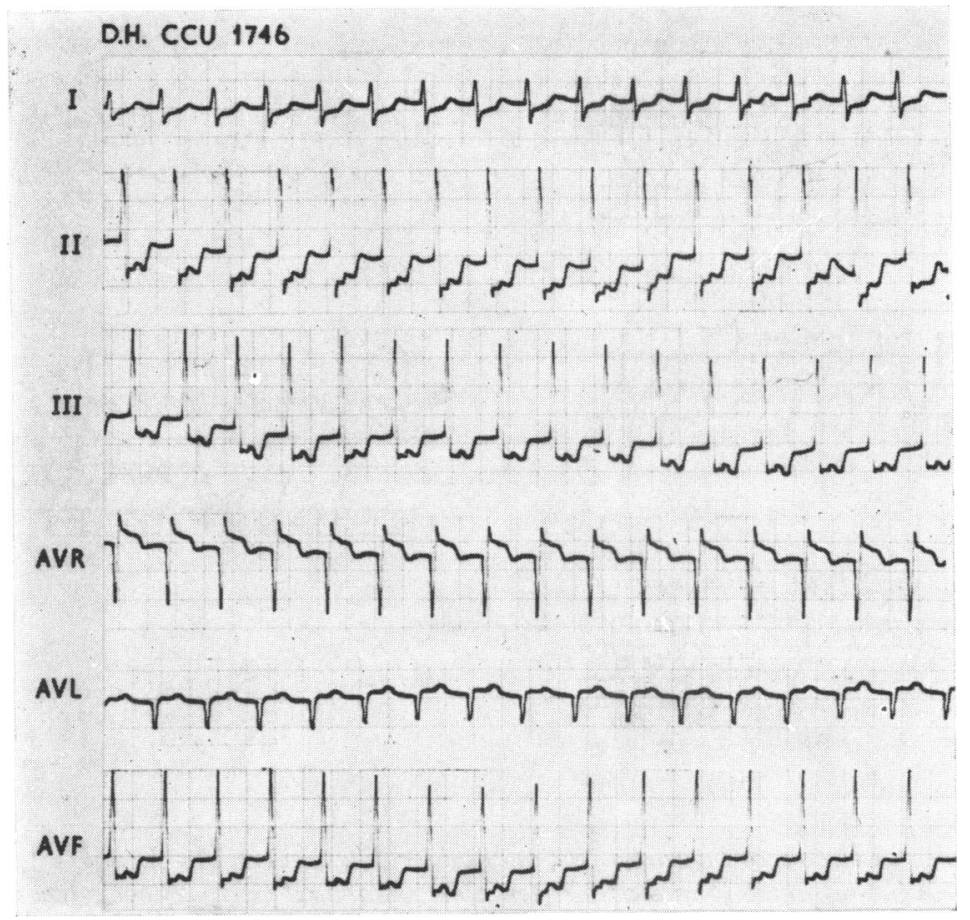

FIG. I Case I, six-lead electrocardiogram during supraventricular tachycardia, before verapamil. Note the normal width $Q R S$ complexes and regularly associated $P$ waves.

the QRS width remained unaltered. The RR interval also alternated from 380 to $480 \mathrm{msec}$, the smaller QRS complexes being preceded by the longer $R R$ interval (Fig. 2). Transient return to sinus rhythm occurred twice before permanent reversion. On both occasions supraventricular tachycardia was precipitated by a ventricular ectopic occurring in late diastole (strips 3,4 , and 5). A single ventricular ectopic which occurred during the tachycardia was not followed by a compensatory pause (strip 5). The $\mathbf{P}$ waves were present but often indistinct in some of the complexes following the taller $\mathbf{R}$ waves, emphasizing the problem of clear delineation of atrial activity when the $P$ waves are embedded in the $T$ waves or ST segment.

The $R R$ interval of the longer cycle increased from 480 to $528 \mathrm{msec}$ immediately before reversion to sinus rhythm. The termination of the tachycardia occurred with a $P$ wave (strip 6) after a total of $4 \mathrm{mg}$ verapamil.

\section{Case 2}

A 20-year-old nurse had suffered three episodes of palpitations in the nine months before admission. Each episode had lasted to to 15 minutes and had reverted spontaneously. Admission was due to an episode which had lasted for four hours. She was not receiving any medication.

On examination the heart rate was 2 ro a minute, blood pressure $85 / 70 \mathrm{mmHg}$, and there was no evidence of cardiac failure. The electrocardiogram showed a supra- ventricular tachycardia. The $Q R S$ complexes were narrow. The $R$ wave sequence occurred in threes and following the small $R$ wave the next two showed progressive increase in amplitude (Fig. 3, strip I). Slight variation in the height of the $Q R S$ complex and the $R R$ interval was also noted in the other QRS complexes (Fig. 3, strip I). No $P$ waves could be identified. Verapamil was given at a rate of $\mathrm{I} \mathrm{mg}$ a minute and after 2 mg (strip 2 and 3 ) every alternate QRS became taller and a clear correlation could be established between the height of the QRS and the preceding RR interval. The latter alternated between 300 and $350 \mathrm{msec}$ and the taller $Q R S$ waves were preceded by the longer $R R$ intervals. After a total of $2.5 \mathrm{mg}$ verapamil, reversion to sinus rhythm occurred and the longer RR interval increased from 350 to $400 \mathrm{msec}$ immediately before reversion to sinus rhythm.

\section{Case 3}

A 43-year-old labourer was admitted with a two-hour history of palpitations. There was no history of cardiac disease. Examination revealed a pulse of 210 a minute, blood pressure $130 / 110 \mathrm{mmHg}$, and absence of cardiac failure. The electrocardiogram showed all QRS complexes to be of normal width and similar amplitude (Fig. 4). No $P$ waves could be identified. The RR interval was regular at $280 \mathrm{msec}$. A diagnosis of supraventricular tachycardia was made and verapamil was admin- 

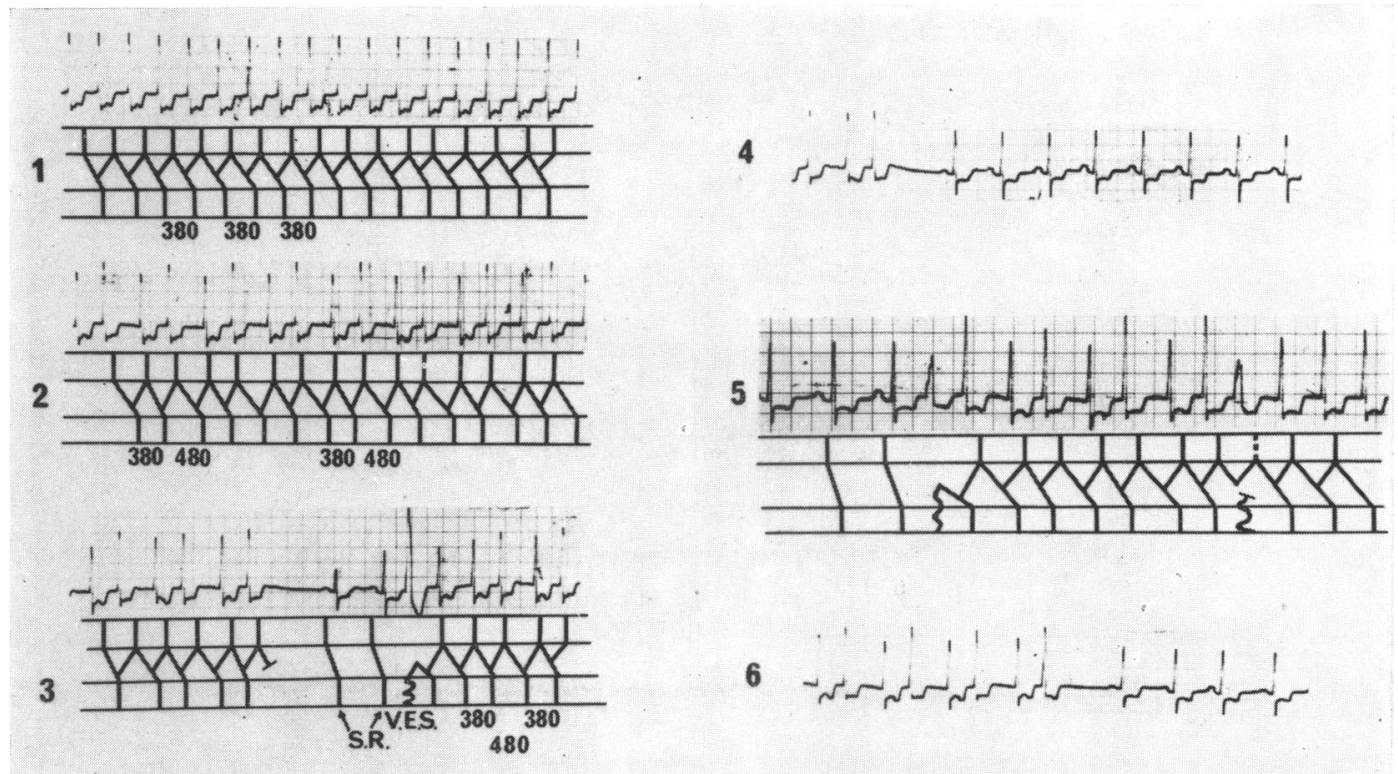

FIG. 2 Selected electrocardiogram strips and ladder diagram ( $A, A=V$ and $V$ ) from Case $I$. Strip I shows a constant $R R$ interval. After $2 \mathrm{mg}$ verapamil (strip 2) cycle length and $Q R S$ amplitude alternation is present. Strips 3 and 4 recorded over the next 2 minutes show transient reversion to sinus rhythm. The arrhythmia recurred after a ventricular ectopic (strip 3). Strip 5 was recorded after $3 \mathrm{mg}$ verapamil and again a ventricular ectopic initiated the arrhythmia. A second ectopic occurring during arrhythmia was not followed by a compensatory pause but the alternation was briefly abolished. Strip 6 was recorded after a total of $4 \mathrm{mg}$ of the drug. The alternation is noted in the first eight beats before reversion to sinus rhythm. The RR interval of the longer cycle increased from 480 to $528 \mathrm{msec}$ (between the 6th and 7 th complex in strip 6). $A P$ wave is seen in the following 8th complex, but was not conducted to the ventricle and sinus rhythm was permanently established. See text for discussion regarding $P$ wave polarity.

istered in doses of $\mathrm{I} \mathrm{mg}$. After a total of $5 \mathrm{mg}$ verapamil, alternation of the $R R$ interval developed with intervals of 288 and 380 msec. There was no change in the QRS configuration or amplitude. No prolongation of the $R R$ interval was seen before reversion to sinus rhythm.

\section{Case 4}

An 18-year-old female patient with bronchial asthma had suffered recurrent episodes of supraventricular tachycardia lasting for several hours and requiring several hospital admissions. She was receiving practolol $200 \mathrm{mg}$ twice daily and bronchodilator therapy.

On examination the heart rate was 160 a minute, blood pressure $95 / 70 \mathrm{mmHg}$. Though she had bilateral rhonchi, she was not in any respiratory distress. The electrocardiogram showed a supraventricular tachycardia with normal width QRS complexes; associated $P$ waves were seen. The $P$ waves, as in Case $I$, were superimposed on the $\mathrm{T}$ waves (strip $\mathrm{r}$, Fig. 5).

She was given intravenous verapamil and alternation of $R R$ cycle length was noted before reversion to sinus rhythm. The QRS morphology remained unchanged. The RR cycle length before verapamil was $380 \mathrm{msec}$; after $3 \mathrm{mg}$ verapamil the alternating $R R$ cycle lengths measured 420 to $540 \mathrm{msec}$ (strip 2). The termination of the tachycardia occurred with a $P$ wave after a total dose of $6 \mathrm{mg}$. The longer RR interval before termination measured 560 msec.

\section{Discussion}

Several workers have suggested that some supraventricular tachycardias are caused by a reciprocating mechanism in the AV nodal pathways (Barker et al., 1943; Bigger and Goldreyer, 1970; Gettes and Yoshonis, 1970). The criteria for the diagnosis of reciprocating tachycardia include the following.

(I) Initiation and termination of tachycardia with an appropriately timed atrial or ventricular ectopic. 


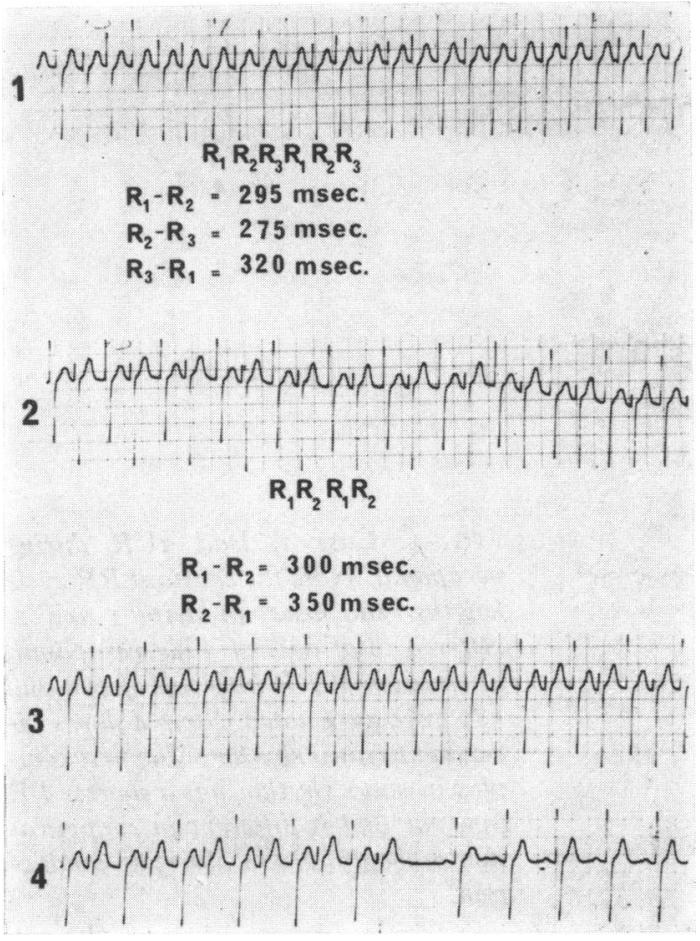

FIG. 3 Case 2, electrocardiogram during verapamil administration. Strip $I$ was recorded before intravenous verapamil. Note the varying $R R$ cycle lengths suggesting a 4:3 Wenckebach block in strip $I$. The amplitude of the QRS varied depending on the cycle lengths. After $2 \mathrm{mg}$ of the drug, alternating $R R$ cycle lengths were noted as shown in strips 2 and 3. The taller $Q R S$ complexes were preceded by longer cycle lengths. Strip 4 was recorded after a total dose of $2.5 \mathrm{mg}$. The first 12 complexes, before reversion to sinus rhythm, show alternation. The RR interval of the longer cycle length preceding reversion (between the Ioth and IIth complex) increased from 350 to 400 msec.

(2) Superiorly directed $\mathbf{P}$ waves which are different from the $\mathbf{P}$ wave which initiates the tachycardia.

(3) Demonstration of atrial or ventricular echoes.

(4) Atrial or ventricular ectopics occurring during tachycardia followed by pauses which are less than compensatory.

In Case I, the initiation of the arrhythmias by an appropriately timed ventricular ectopic and the absence of a compensatory pause following a ventricular ectopic beat, occurring during the tachycardia, suggest a reciprocating mechanism. Gettes and Yoshonis (1970) suggested that the diagnosis of reciprocating tachycardia should be suspected when the arrhythmia recurs within minutes of termination. In this patient the arrhythmia recurred on three occasions after a transient return to sinus rhythm. The reciprocating mechanism in the AV node implies that the atria are retrogradely excited and the $P$ wave axis should be superiorly directed. Massumi and Desando (1972) have shown, however, that the estimation of $P$ wave axis can be misleading when the $P$ waves fall in the $S T$ segment or are superimposed on the $T$ waves, and intra-atrial or oesophageal leads are necessary for elucidation of $\mathbf{P}$ axis under these circumstances.

Spurrell et al. (1973) reported alternating cycle lengths in two of three patients (Cases $I$ and 3 ) with induced reciprocating tachycardia. The authors postulated three pathways, one for retrograde conduction and two for antegrade conduction. The alternation was explained on the basis of differing conduction in the antegrade pathways.

In our first patient, initially the RR cycle length was constant, and the alternation of $R R$ cycle lengths only occurred after the administration of verapamil. One explanation is that the drug produced a 2:I block in the antegrade pathway unmasking a further intranodal pathway for antegrade conduction with a change in sequence of the activation of the ventricle (Fig. 6). It is, however, possible that the resultant change in the QRS morphology was due to unmasking of an accessory, rather than an intranodal, pathway. This $2: \mathrm{I}$ block in the antegrade pathway would explain the fact that the short $R R$ interval ( $380 \mathrm{msec}$ ) during alternating cycle lengths was the same as the $R R$ interval before alternation. The $R R$ interval of the longer cycles was $480 \mathrm{msec}$ and increased to $528 \mathrm{msec}$ immediately before reversion, suggesting that the termination of the arrhythmia resulted from increased conduction time of the unmasked antegrade pathway brought about by higher doses of verapamil. The alternating $R R$ cycles were also associated with changing QRS amplitude and suggested a changing sequence of ventricular depolarization caused by anomalous conduction. Spurrell et al. (1973) have reported unmasking of a retrograde Kent pathway after administration of intravenous verapamil. In our patient the drug appeared to unmask an antegrade pathway.

Another possible explanation for alternating cycle lengths in a reciprocating supraventricular tachycardia deserves mention. A drug-induced $3: 2$ Wenckebach block occurring simultaneously in the initial common and final pathway could explain alternation, but it implies almost equal refractoriness and conduction in these pathways and appears 


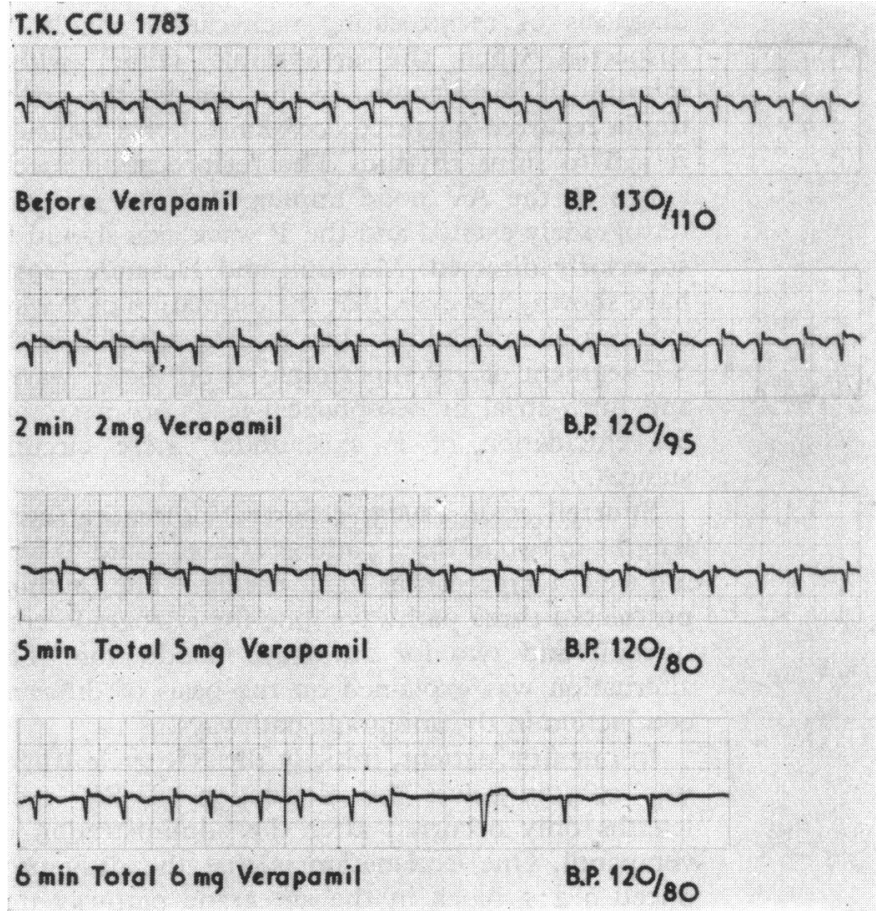

FIG. 4 Case 3, lead aVR during verapamil. Note the constant $R R$ cycle lengths (280 msec) in strips $I$ and 2. After a total dose of $5 \mathrm{mg}$ verapamil, alternation of $R R$ intervals of 288 and $380 \mathrm{msec}$ were noted. Strip 4 shows reversion to sinus rhythm. The first complex in sinus rhythm has a shorter $P R$ interval and is different in configuration probably due to aberrant conduction.

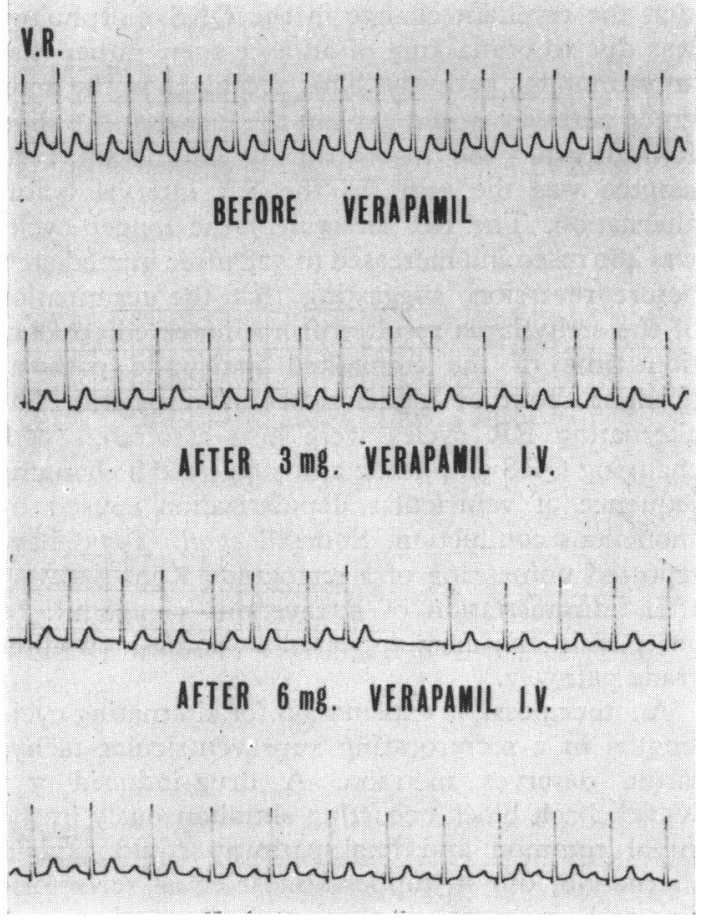

FIG. 5 Case 4: The first strip shows lead II during tachycardia with a constant $R R$ cycle length of 380 msec. After $3 \mathrm{mg}$ verapamil, alternating $R R$ cycle lengths measured 420 and $540 \mathrm{msec}$. Reversion to sinus rhythm occurred after $6 \mathrm{mg}$ of the drug. The $P$ waves are seen embedded in the $S T$ segment during tachycardia. 
unlikely on the basis of experimental studies (Watanabe and Dreifus, I965).

It is not possible to offer a definite single mechanism for the alternation of $R R$ cycle lengths in the remaining three patients.

In Case 2, a 4:3 Wenckebach block was present before the use of verapamil. Alternation of cycle lengths in this case can be explained on the basis of progression of $4: 3$ to a 3:2 Wenckebach block in an atrial or AV junctional extrasystolic tachycardia (Fig. 7). Though the exact mechanism for the changing QRS morphology remains uncertain, it may be attributed to the increased refractory period after longer cycle lengths. However, this can also be explained on the basis of a reciprocating tachycardia with a 4:3 Wenckebach block in the final common pathway which progressed to a 3:2 Wenckebach after verapamil with resulting cycle length alternation (Fig. 7), the aberration in QRS conduction occurring more distally.

Cases 3 and 4 differ from Cases $I$ and 2 in that the QRS morphology remained unaltered despite the changing RR intervals. These could be ex-
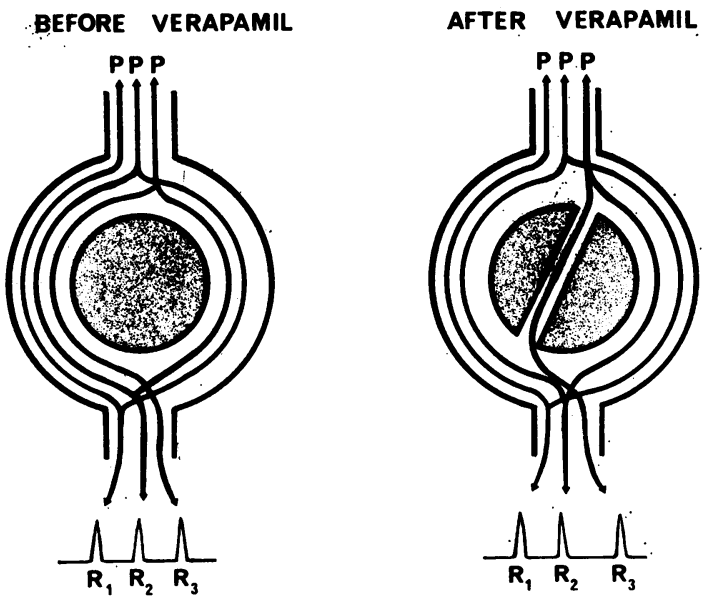

FIG. 6 Two pathways, antegrade (conduction time $285 \mathrm{msec}$ ) and retrograde (conduction time $95 \mathrm{msec}$ ) before verapamil, suggested opening up of a second pathway for antegrade conduction (385 msec) caused by a 2:I block in the original antegrade pathway. The retrograde pathway is unaltered.
BEFORE VERAPAMIL
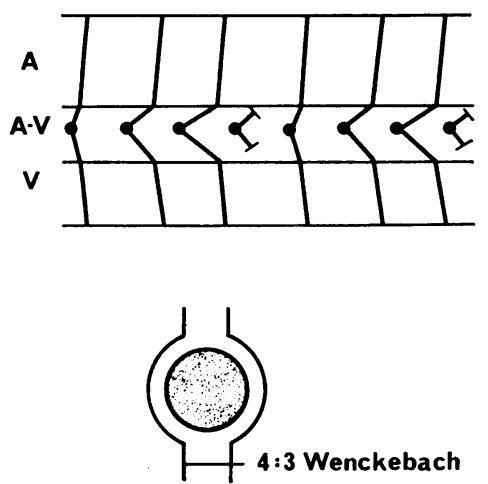

AFTER VERAPAMIL
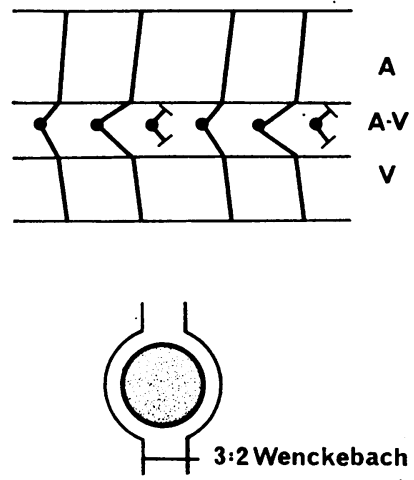

FIG. 7 The suggested mechanisms of alternation in Case 2. The ladder diagram shows progression from 4:3 to 3:2 Wenckebach block in an extrasystolic $A V$ junctional tachycardia resulting in alternation. The lower diagram suggests the possible mechanism of alternation in this patient on the basis of a reciprocating tachycardia. See text for discussion.

amples of extrasystolic atrial or nodal tachycardia with drug-induced 3:2 Wenckebach block, described as 'pseudo alternation' by Langendorf (1958) or a reciprocating tachycardia with alternation as a result of an unmasked antegrade pathway as in Case I.

Schamroth et al. (1972) have also reported Wenckebach block in response to verapamil in two patients with supraventricular tachycardia, but this occurred just before the termination of the arrhythmias and did not result in alternating cycle lengths.

We are grateful to Dr H. J. L. Marriot for his comments on the electrocardiograms reported here.

\section{References}

Barker, P. S., Johnston, F. D., and Wilson, F. N. (1943). Auricular paroxysmal tachycardia with alternation of cycle length. American Heart fournal, 25, 799. 
Bigger, J. T., and Goldreyer, B. N. (1970). The mechanism of supraventricular tachycardia. Circulation, 42, 673.

Gettes, L. S., and Yoshonis, K. F. (1970). Rapidly recurring supraventricular tachycardia. Circulation, 41, 689.

Katz, L. N., and Pick, A. (1960). Current status of theories of mechanisms of atrial tachycardias, flutter and fibrillation. Progress in Cardiovascular Diseases, 2, 650.

Langendorf, R. (1958). Alternation of A-V conduction time. American Heart fournal, 55, I81.

Massumi, R. A., and Desando, C. J. (1972). Intra-atrial and esophageal electrography in the diagnosis of complex arrhythmias. In Advances in Electrocardiography, p. 229. Ed. by R. C. Schlant and J. W. Hurst. Grune and Stratton, New York and London.

Mendez, C., and Moe, G. K. (1966). Demonstration of a dual A-V nodal conduction system in the isolated rabbit heart. Circulation Research, 19, 378.

Moe, G. K., Childers, R. W., and Merideth, J. (1968). An appraisal of 'supernormal' A-V conduction. Circulation, 38, 5 .
Schamroth, L., Krikler, D. M., and Garrett, C. (1972). Immediate effects of intravenous verapamil in cardiac arrhythmias. British Medical fournal, 1, 660.

Schuilenberg, R. M., and Durrer, D. (1968). Atrial echo beats in the human heart elicited by induced atrial premature beats. Circulation, 37, 680 .

Spurrell, R. A. J., Krikler, D., and Sowton, E. (1973). Two or more intra AV nodal pathways in association with either a James or Kent extranodal bypass in 3 patients with paroxysmal supraventricular tachycardia. British Heart fournal, 35, II3.

Watanabe, Y., and Dreifus, L. S. (1965). Inhomogenous conduction in the A-V node. American Heart fournal, 70, 505.

Requests for reprints to Dr. J. K. Vohra, Cardiac Department, The Royal Melbourne Hospital, 3050 Victoria, Australia. 